\title{
Internet-mediated physiotherapy and pain coping skills training for people with persistent knee pain (IMPACT - knee pain): a randomised controlled trial protocol
}

Fiona Dobson ${ }^{1}$, Rana S Hinman', Simon French ${ }^{1,2}$, Christine Rini ${ }^{3}$, Francis Keefe ${ }^{4}$, Rachel Nelligan ${ }^{1}$, $J_{\text {J Haxby Abbott }}^{5}$, Christina Bryant ${ }^{6}$, Margaret P Staples ${ }^{7}$, Andrew Dalwood ${ }^{8}$ and Kim L Bennell ${ }^{1^{*}}$

\begin{abstract}
Background: Persistent knee pain in people over 50 years of age is often attributable to knee osteoarthritis (OA), a common joint condition that causes physical and psychological dysfunction. Exercise and pain coping skills training (PCST) can help reduce the impact of persistent knee pain, however, access to health professionals who deliver these services can be challenging. With increasing access to the Internet, remotely delivered Internet-based treatment approaches may provide alternatives for healthcare delivery. This pragmatic randomised controlled trial will investigate whether an Internet-delivered intervention that combines PCST and physiotherapist-guided exercise (PCST + Ex) is more effective than online educational material (educational control) in people with persistent knee pain.

Methods/Design: We will recruit 148 people over 50 years of age with self-reported persistent knee pain consistent with knee OA from the Australian community. Following completion of baseline questionnaires, participants will be randomly allocated to access a 3-month intervention of either (i) online educational material, or (ii) the same online material plus an 8-module (once per week) Internet-based PCST program and seven Internet-delivered physiotherapy sessions with a home exercise programs to be performed 3 times per week. Outcomes will be measured at baseline, 3 months and 9 months with the primary time point at 3 months. Primary outcomes are average knee pain on walking (11-point numeric rating scale) and self-reported physical function (Western Ontario and McMaster Universities Osteoarthritis Index subscale). Secondary outcomes include additional measures of knee pain, health-related quality-of-life, perceived global change in symptoms, and potential moderators and mediators of outcomes including self-efficacy for pain management and function, pain coping attempts and pain catastrophising. Other measures of adherence, adverse events, harms, use of health services/co-interventions, and process measures including appropriateness and satisfaction of the intervention, will be collected at 3, 6 and 9 months.
\end{abstract}

Discussion: The findings will help determine the effectiveness and acceptability of Internet access to a combination of interventions that are known to be beneficial to people with persistent knee pain. This study has the potential to guide clinical practice towards innovative modes of healthcare provision.

Trial registration: Australian New Zealand Clinical Trials Registry reference: ACTRN12614000243617.

Keywords: Knee osteoarthritis, Knee pain, Physiotherapy, Pain coping skills, Internet, Health care delivery, Randomised control trial

\footnotetext{
*Correspondence: k.bennell@unimelb.edu.au

${ }^{1}$ Centre for Health, Exercise and Sports Medicine, Department of

Physiotherapy, School of Health Sciences, The University of Melbourne, Alan

Gilbert Building 161 Barry St, Carlton, Vic 3053, Australia

Full list of author information is available at the end of the article
} 


\section{Background}

Osteoarthritis (OA) is the leading cause of persistent knee pain in people over 50 years old [1]. In the Global Burden of Disease 2010 study [2], knee OA, together with hip OA, was ranked as the eleventh highest contributor to global disability with a global age-standardised prevalence of knee OA estimated at 3.8\% [3]. At the individual level, persistent pain due to knee OA can be debilitating. It leads to loss of function, reduced quality of life [4], and psychological disability [5]. Many people with knee OA experience co-morbidities such as obesity, depression and cardiovascular disease that add further burden to the disease. With an aging and increasingly obese world population, that are projected to increase the prevalence of knee OA pain, there is an urgent need for accessible and effective treatments that improve patient symptoms and function, while minimising the costs to both patients and society.

Interventions that foster self-management, such as exercise, are considered fundamental to managing knee pain due to knee OA. Considerable evidence supports the use of strengthening exercises to reduce pain and improve function in this patient population [6-10]. Exercise is universally recommended by clinical guidelines [11] regardless of age, disease severity, pain intensity, functional levels and co-morbidities. Meta-analyses [12,13] consistently report benefits of all types of exercise compared to education or no treatment and exercise programs that combine strengthening, flexibility and aerobic exercise seem to provide the best benefits for improving pain and function [14]. Importantly, exercise is regarded as a safe intervention in patients with knee OA with few contraindications or adverse events [15].

Although regular exercise can reduce physical impairments and improve participation in everyday life activities [16], meta-analysis has consistently revealed it produces relatively modest improvements in pain and function $[13,14,17]$. Furthermore, some patients experience increased pain when they start exercising leading them to discontinue this treatment. Exercise primarily targets biomedical factors that can influence pain and does not directly address psychological factors such as self-efficacy for pain control, adaptive pain coping skills and overly negative thinking about pain (pain catastrophising). As low self-efficacy and ineffective pain coping strategies are common in chronic pain populations $[5,18,19]$, interventions that target these key psychological factors in addition to biomedical factors may provide more powerful benefit. Accordingly and consistent with a biopsychosocial approach to chronic pain management, there has been growing interest in interventions that combine exercise and psychological treatments.

Cognitive behavioural therapy is the most frequently studied psychological intervention for pain control in individuals with arthritis [20]. Growing evidence supports the use of pain coping skills training (PCST), an approach based on cognitive behavioural principles, to improve pain and psychological functioning in chronic pain conditions [21-23]. More recent evidence shows that PCST combined with exercise, delivered by specially trained physiotherapists, is effective in people with knee pain due to knee OA [24]. Despite the advantages of using an intervention that combines exercise and PCST, access to specialised health professionals to deliver this combined intervention is not readily available. As PCST is currently in the domain of specialised psychologists, people with OA knee pain are not always able to access specialised health professionals to deliver this combined intervention, especially if they live in rural and remote areas $[25,26]$.

The Internet has been increasingly used as a time efficient and convenient method to deliver health interventions [27]. It is estimated that around 34\% of the world's population (roughly 2.4 billion people) use the Internet [28]. Its use is growing in all segments of our society, including within the home, where there has been a 566\% increase in worldwide use over the last decade [28]. Interestingly, older adults are the age group with the fastest growing Internet usage [29]. Therefore, the Internet represents a viable delivery mode to allow interventions to reach a large number of individuals, including older adults living in remote areas.

Members of our research team (CR, FK) recently developed an automated, Internet-based PCST program called PainCOACH to improve access to PCST for people with persistent OA pain. This program is likely to be more cost effective than face-to-face PCST because it eliminates the need for trained professionals to lead weekly face-to-face sessions [30-33]. It also has the potential to be more convenient than face-to-face PCST because people with OA can use it at home at a time that suits them. Similarly, teleconferencing and Internetbased telerehabilitation have been used as alternative methods for delivering physiotherapy and exercise [34]. These methods have been found to be useful for people with persistent knee pain [35] and are comparable to conventional face-to-face physiotherapy for people following knee joint replacements [36,37]. Furthermore, both health professionals and patients using Internetbased methods reported levels of satisfaction that were no different than those reported by health professionals and patients using traditional consultation methods [38]. However, the combination of delivering both PCST and physiotherapy over the Internet has not yet been rigorously evaluated in people with persistent knee pain consistent with knee OA.

The primary objective of this pragmatic randomised controlled trial (RCT) is to evaluate whether an Internet-based 
intervention that combines PCST and physiotherapistguided exercise (PCST $+\mathrm{Ex})$, is more effective than online educational material (educational control) in people with persistent OA knee pain. We hypothesise that PCST + Ex will be more effective in reducing pain and improving selfreported physical function after 3 months than the educational control intervention. Secondary hypotheses are (i) the PCST + Ex will be more effective in reducing pain and improving self-reported physical function after 9 months than the educational control intervention; and (ii) there will be greater improvements in health-related quality of life and psychological outcomes, as well as a better participant- perceived response to treatment in the PCST $+\mathrm{Ex}$ after 3 months and 9 months than the education control intervention.

\section{Methods/Design}

\section{Trial design}

Parallel-design 2-arm pragmatic RCT, with a 3-month intervention and outcomes assessed at 3 and 9 months from baseline. The primary outcome time point will be 3 months. Reporting of the trial will comply with the CONSORT statement for randomised trials and the extension for trials assessing non-pharmacologic treatments [39,40] (Figure 1).

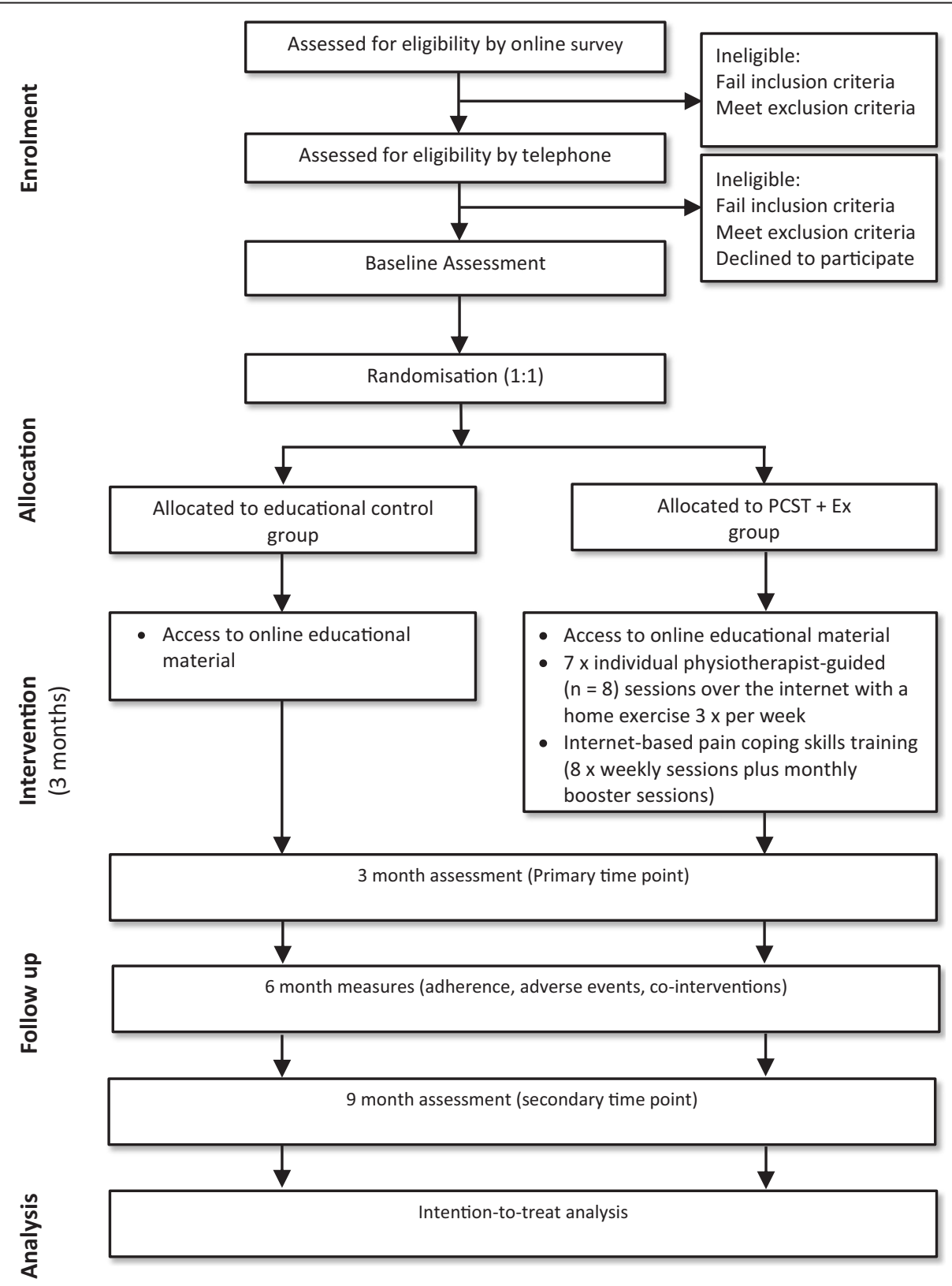

Figure 1 Flow diagram of study protocol. 


\section{Participants}

We will recruit 148 participants aged $\geq 50$ years with persistent OA knee pain from the Australian community via advertisements, media campaigns, social media (e.g. Facebook and Twitter), university websites, our research volunteer database, and other external databases of volunteers who have registered as being interested in participating in clinical research. Participants will be included if they i) are aged $\geq 50$ years; ii) report persistent knee pain for more than 3 months and for most days of the past month; iii) report a minimum average knee pain intensity during walking of 4 on an 11-point numerical rating scale (NRS, with terminal descriptors of 'no pain' and 'worst pain possible') in the past week; iv) report at least mildmoderate physical dysfunction (score $>20$ on the Western Ontario McMaster Universities (WOMAC) physical function subscale); and v) have an active email account and access to a computer with a broadband Internet connection.

Participants will not be eligible if they: i) have had a knee joint replacement on the painful knee side; ii) are on the waiting list for joint replacement surgery; iii) have undergone intra-articular corticosteroid injection or knee surgery to either knee within past 6 months or are planning joint surgery in the next 9 months; iv) are currently receiving or have received treatment for knee pain (e.g. physiotherapy, chiropractic, osteopathy, acupuncture or psychologist)in the last six months from a health practitioner, or if they have participated in a muscle strengthening exercise program or a pain coping skills program in the last 6 months; v) have a systemic arthritic condition; vi) have any neurological conditions that affect the lower-limb and limit their ability to exercise safely (such as a stroke, multiple sclerosis, polio, neuropathy, peripheral nerve disease or Parkinson's disease); vii) have any other major joint pain (e.g. back, hip or ankle) to a greater extent than their knee pain that currently limits the ability to exercise; viii) have selfreported high-level depression (score of $>21$ on the depression subscale of the Depression, Anxiety and Stress Scale) and/or; ix) are not fluent in written and spoken English.

\section{Procedure}

Figure 1 outlines the trial phases. Eligibility of volunteers will be initially confirmed via an online screening survey followed by a telephone interview. Baseline, 3-month (primary time point) and 9-month (secondary time point) assessments will be conducted via the Internet using online assessment surveys. Additionally, participants will complete log books to capture adherence and adverse event data. These will be either transcribed by participants to an online survey or mailed back to researchers, as preferred by each participant. Ethical approval has been obtained from the University of Melbourne Human
Research Ethics Committee (No. 1339459). All participants will provide written informed consent. For participants with bilaterally eligible knees, only the most painful knee (as identified by the participant) will be evaluated.

\section{Randomisation and allocation concealment}

On completion of baseline assessment, participants will be consecutively randomised to one of two groups: 1) educational control (online educational material) or 2) intervention group (online educational material, Internetbased PCST and physiotherapy-guided exercise program delivered via the Internet). Computer generated randomisation will be conducted by random permuted blocks of size 2-8 stratified by gender (male or female) and residency (metropolitan or rural), prepared by our study biostatistician (MS). To conceal randomization, consecutively numbered, sealed, opaque envelopes will be prepared by a researcher with no other involvement in the study. The envelopes will be stored in a locked location and will be opened in sequence within each stratum to reveal group allocation.

All primary and secondary outcome data will be completed online and thus will be automatically entered into a database. By necessity, participants and physiotherapists will be unblinded to group allocation; however, participants will remain blinded to research hypotheses. The statistician will be blinded to group allocation until completion of the statistical analyses.

\section{Interventions}

\section{Education control condition}

Participants allocated to the educational control group will receive a specific URL-link to access online educational material about knee OA. This educational material comprises information sheets produced and provided by Arthritis Australia that is also available to the general public via their website (www.arthritisaustralia.com.au). It will contain a recommended reading list that covers topics such as exercise and physical activity, managing pain, and general information about knee pain and knee OA. It will also contain an additional reading list that will cover topics such as emotions, healthy eating, and complementary therapies and medications. Participants in the control group are encouraged to access the education material at their own leisure and pace.

\section{PCST + Ex condition}

Participants in the PCST + Ex group will receive three interrelated interventions that are delivered via the Internet. First, they will receive the same specific URL-link to access the same online educational material as the educational control group. Second, they will receive access to PainCOACH, which includes eight 35- to 45-minute modules that each 
teach a pain coping skill shown to help people manage persistent OA pain. The modules are designed to be completed at the rate of one per week. Participants will also receive seven physiotherapy consultations delivered over the Internet using a video telephony service, Skype ${ }^{\mathrm{Tu}}$. The initial session will be 45 -minutes, followed by 30-minute sessions thereafter. During these consultations, participants will be prescribed an individualised home-based exercise program to be performed three times weekly. The weekly Pain$\mathrm{COACH}$ sessions will occur during the 3-month intervention period in Weeks 1-8, with a booster session at Week 11. The physiotherapy sessions will occur in Weeks 2, 3, 4, $6,8,10$ and 12 of the 3-month intervention period. Participants will receive further monthly PainCOACH boosters during the 6 months following the intervention period, where they will be encouraged to review the final module of the program as well as revisit useful/meaningful modules. They will also receive monthly email reminders to encourage them to continue their exercises at home and practice their pain coping skills.

Prior to beginning the intervention, participants will receive Skype set-up training, including instructions on how to set up their web-camera and their exercise environment. They will also be given an instructional manual and password access to PainCOACH from the researchers. Any equipment required for the exercises (e.g. elastic bands, ankle weights, web camera if required, and pedometer if required) will be mailed to participants before the intervention commences.

The PCST component will be delivered using the Pain$\mathrm{COACH}$ program, which translates key therapeutic components of face-to-face PCST [41] for delivery in eight highly interactive, automated training sessions (i.e., it does not require interaction with a therapist). The program's eight modules provide interactive training in a cognitive or behavioural pain coping skill. Module 1 provides an overview describing the PainCOACH program, PCST, and a therapeutic rationale to explain how pain coping skills can help people manage OA pain [42]. This overview is followed by training in the first pain coping skill: progressive muscle relaxation. Modules 2-7 teach brief relaxation skills, activity-rest cycling, pleasant activity scheduling, cognitive restructuring, pleasant imagery, and problem solving. Module 8 consolidates learning and teaches strategies for long term skill use. A summary of the content and flow of PainCOACH modules is provided in Table 1. Consistent with a face-to-face PCST protocol, participants are asked to practice each new skill they learn. For each new skill, participants' completion of practices and experiences with them are reviewed at the beginning of the subsequent week's module.

A variety of program features were designed to enhance engagement and adherence [43]. For instance, participants are led through the program by a female virtual "coach" who speaks directly to them as she provides verbal instruction, feedback, and encouragement. Her dialogue is accentuated with changing images (e.g., illustrative graphics or pictures of the coach that change so that her body language and facial features correspond to ideas being expressed) and onscreen text highlighting key ideas. In addition, theoretically-based methods drawn from social cognitive theory $[44,45]$, adult learning theory $[46,47]$, and principles of multimedia instruction [e.g., 48] are used to reinforce skill learning and mastery. The overall approach is consistent with research suggesting that certain combinations of text, audio, animation, and graphics can enhance learning e.g., [48].

In addition, PainCOACH includes supplementary features to support use of new skills. For instance, $\mathrm{COACH}$ track promotes self-monitoring by allowing participants to review and change practice goals, record practices and "coping confidence" (self-efficacy), view graphic summaries of their progress over time, and manage automated practice reminders. Another feature, called $\mathrm{COACH}-$ chat, allows them to read about others' experiences using PCST and to submit descriptions of their own experiences. It was designed to provide benefits of observational learning that people get in face-to-face PCST groups. Finally, $M y C O A C H$ provides information about the program, the study, and actions to take in a medical or mental health emergency. A companion workbook provides instructions for using PainCOACH, an overview of its modules and features, and worksheets and practice logs.

Finally, participants allocated to the intervention group will receive a physiotherapist-guided home-based exercise program primarily designed to strengthen lower limb muscles. It will be progressed over the program to maintain a moderate intensity. Exercises will be prescribed based on our own previous exercise program used for face-to-face interventions in people with knee OA [49]. They have been shown to improve pain and physical function [50] and reflect current clinical practice (Table 2). Participants will be prescribed a minimum of five and a maximum of six exercises. These will include two knee extensor strengthening exercises, one each of a hip abductor, hamstring, and calf strengthening exercise and one additional exercise (from a limited list) chosen by the physiotherapist based on assessment. Participants will be directed to online instructions and video demonstrations of the home exercises to help guide the exercises set by the physiotherapist. They will also be encouraged to increase their overall level of physical activity and will receive written information about ways to do so as well as the option of using a pedometer as a motivational tool.

Using the Internet to mediate learning, the physiotherapist will begin each participant's exercise program by selecting exercises and prescribing dosages based on 
Table 1 Summary of the internet-based PainCOACH content

\begin{tabular}{|c|c|c|}
\hline $\begin{array}{l}\text { Module } \\
\text { number }\end{array}$ & Coping skill & Content \\
\hline 1 & Progressive relaxation & $\begin{array}{l}\text { Teach Gate Control Theory (how thoughts, feelings, and actions affect and are affected } \\
\text { by pain). Introduce and demonstrate progressive relaxation with animation; walk user } \\
\text { through use of the technique and active practice; help user identify/address } \\
\text { circumstances that might impede relaxation and chose strategies to overcome } \\
\text { obstacles; plan regular practice times; set practice goal. }\end{array}$ \\
\hline 2 & Mini-practices & $\begin{array}{l}\text { Review prior session content and practices; Introduce and demonstrate "mini-practices" } \\
\text { (brief relaxation) with animation; walk user through use of the technique and active practice, } \\
\text { gather/evaluate pre- and post- activity pain; help user identify/address circumstances that } \\
\text { might impede relaxation and chose strategies to overcome obstacles; discuss benefits and } \\
\text { reminders for practicing; plan regular practice times; set and review practice goals. }\end{array}$ \\
\hline 3 & Activity/rest cycling & $\begin{array}{l}\text { Review prior session content and practices; Introduce concept of activity/rest cycling; identify } \\
\text { activities user tends to overdo; vicarious learning exercise demonstrate how to change } \\
\text { overdone activities; create personal plan to fit daily routine and personal goals; review how } \\
\text { other skills help with use of this one; plan regular practice times; set and review practice } \\
\text { goals. }\end{array}$ \\
\hline 4 & $\begin{array}{l}\text { Pleasant activity scheduling and identify } \\
\text { negative automatic thoughts }\end{array}$ & $\begin{array}{l}\text { Review prior session content and practices; Introduce concept of pleasant activity scheduling; } \\
\text { lead user through exercise for adding pleasant activities to their lives; mini-practice of } \\
\text { 10-minute pleasant activity to be done immediately (gather/ evaluate pre- and post- activity } \\
\text { pain); schedule } 3 \text { pleasant activities for week; problem-solve barriers with interactive vicarious } \\
\text { learning exercise; Introduce concept of negative automatic thoughts; describe connections } \\
\text { between thoughts, emotions, behaviors, and pain; walk user through a thoughts exercise; } \\
\text { plan regular practices; set and review practice goals. }\end{array}$ \\
\hline 5 & $\begin{array}{l}\text { Identify/change negative automatic } \\
\text { thoughts and coping thoughts }\end{array}$ & $\begin{array}{l}\text { Review prior session content and practices; Continue and advance prior session's activities } \\
\text { related to automatic thoughts and introduce coping thoughts. Practice identifying negative } \\
\text { thoughts and accompanying emotional and physical reactions of virtual patients, then self; } \\
\text { exercise to teach generation of alternative thoughts, then practice and record accompanying } \\
\text { sensations. Focus on teaching generation of alternative thoughts, practice generating } \\
\text { calming self-statements; practice skills and get feedback; identify and address circumstances } \\
\text { that impede use of these skills and strategies to overcome obstacles; "mini-practices" for } \\
\text { specific circumstances; plan regular practices; set and review practice goals. }\end{array}$ \\
\hline 6 & $\begin{array}{l}\text { Pleasant imagery and distraction } \\
\text { techniques }\end{array}$ & $\begin{array}{l}\text { Review prior session content and practices; Introduce pleasant imagery and auditory and } \\
\text { focal point distraction techniques; complete exercises with audio instructions; plan regular } \\
\text { practices; set and review practice goals. }\end{array}$ \\
\hline 7 & Problem Solving & $\begin{array}{l}\text { Review prior session content and practices; Introduce concept of problem solving and } \\
\text { describe steps; demonstrate problem solving with character stories; generate list of } \\
\text { challenging situations; exercise to help users select skills for each situation, with personalized } \\
\text { plan for overcoming barriers; plan regular practices; set and review practice goals. }\end{array}$ \\
\hline \multirow[t]{2}{*}{8} & Monitoring for maintenance & $\begin{array}{l}\text { Review all session content; evaluate skill frequency, helpfulness and comparison to other } \\
\text { users; exercises to develop plan for maintenance of skills; motivate further practice and skill } \\
\text { development; remind how skills facilitate personal goals; review practice goals. }\end{array}$ \\
\hline & Booster sessions & Review module 8 as well as revisit any useful/meaningful sessions. \\
\hline
\end{tabular}

findings from an initial assessment of the participant's pain and perceived level of effort during performance of an exercise. The participant will learn each exercise by observing as the physiotherapist demonstrates the exercise and then performing it under the physiotherapist's supervision.

The physiotherapist will perform a brief assessment at each physiotherapy session to ascertain any adverse effects that may have occurred with home exercises and check quality and form of exercise performance. Progression of exercises will be provided by varying the type of exercise as well as the number of repetitions, load or degree of difficulty within an exercise. The prescribed resistance will aim to approximate a 10-repetition maximum level and a patient-rated level of effort experienced during each strengthening exercise of at least 5 out of 10 (hard) on a modified Borg Rating of Perceived Exertion CR-10 scale designed specifically for strengthening exercise [51].

In order to minimise burden of exercise, only the study knee will be the focus of treatment and evaluation. If participants have bilateral symptoms, the physiotherapist may choose exercises that are performed in weight-bearing on both legs simultaneously to achieve bilateral strength gains within the constraints of the treatment protocol.

Although some discomfort is expected during the exercise intervention, any exercise-related pain should subside to usual levels by the next day with no increase in knee swelling following the exercise session. Participants 
Table 2 Home exercise program protocol Maximum of 6 exercises (with progression as appropriate)
2 knee extensor strengthening exercises
1 hip abductor strengthening exercise
1 hamstring strengthening exercise
1 calf strengthening exercise
1 other exercise chosen based on assessment findings

\section{Quads strengthening (each program must include 2 exercises)}

\begin{tabular}{|c|c|c|c|}
\hline \multirow[t]{6}{*}{ Knee extension } & \multirow[t]{3}{*}{ Non weight-bearing } & \multirow{3}{*}{$\begin{array}{l}\text { A. Seated knee extension } \\
\text { (with resistance) with } \\
5 \text { second hold }\end{array}$} & Indications: suggested as an initial exercise \\
\hline & & & $\begin{array}{l}\text { Progression: Increase cuff weight or theraband resistance - red } \\
\text { through to black }\end{array}$ \\
\hline & & & Simplification: eliminate weight or see $1 \mathrm{~B}$ \\
\hline & \multirow[t]{3}{*}{ Non weight-bearing } & \multirow{3}{*}{$\begin{array}{l}\text { B. Inner range quads over } \\
\text { roll (with resistance) with } \\
5 \text { second hold }\end{array}$} & $\begin{array}{l}\text { Indications: Usually only required when any flair ups with seated } \\
\text { knee extension (1A) }\end{array}$ \\
\hline & & & Progression: Use appropriate level of ankle cuff weight \\
\hline & & & Simplification: eliminate weight if flare up \\
\hline \multirow[t]{3}{*}{ Sit-to-stand } & \multirow[t]{3}{*}{ Weight-bearing } & \multirow{3}{*}{$\begin{array}{l}\text { C. Sit to stand without } \\
\text { using hands }\end{array}$} & Indications: suggested as an initial exercise \\
\hline & & & $\begin{array}{l}\text { Progression: lower chair height, hover above the seat without } \\
\text { touching down, more weight on affected leg, slit leg position } \\
\text { (affected leg closer to seat) }\end{array}$ \\
\hline & & & Simplification: use hands \\
\hline \multirow[t]{6}{*}{ Steps } & \multirow[t]{3}{*}{ Weight-bearing } & \multirow[t]{3}{*}{ D. Step-ups } & Indications: suitable progression from sit to stand (1C) \\
\hline & & & $\begin{array}{l}\text { Progression: Increase step height, hold extra weight (in hands } \\
\text { or backpack) }\end{array}$ \\
\hline & & & Simplification: sit to stand (1C) \\
\hline & \multirow[t]{3}{*}{ Weight-bearing } & \multirow{3}{*}{$\begin{array}{l}\text { E. Forward touchdowns } \\
\text { from a step }\end{array}$} & Indications: suitable progression from step-ups (1D) \\
\hline & & & $\begin{array}{l}\text { Progression: Increase step height, hold extra weight (in hands } \\
\text { or backpack), don't touch down }\end{array}$ \\
\hline & & & Simplification: step-ups (1D) \\
\hline \multirow[t]{3}{*}{ Partial squats } & \multirow[t]{3}{*}{ Weight-bearing } & \multirow[t]{3}{*}{ F. Partial wall squats } & Indications: suitable progression from sit to stand (1C) \\
\hline & & & Progression: Increase to $5 \mathrm{sec}$ hold, more weight on study side) \\
\hline & & & $\begin{array}{l}\text { Simplification: if find flare/problematic step back to sit to } \\
\text { stand }(1 C)\end{array}$ \\
\hline
\end{tabular}

\section{Hip abductor strengthening (1 exercise)}

Standing hip abduction Non weight-bearing A. Side leg raises in standing Indications: suggested as an initial exercise

Progression: Increase cuff weight or theraband resistance - red through to black

Simplification: eliminate weight

\begin{tabular}{llll}
\hline Side stepping & Weight-bearing & $\begin{array}{l}\text { B. Crab walk with resistance } \\
\text { band }\end{array}$ & $\begin{array}{l}\text { Indications: good progression from standing leg side raises (2A) } \\
\text { Progression: Increase theraband resistance - red through to black } \\
\text { Simplification: side leg raises in standing (2A) }\end{array}$ \\
\hline Standing hip abduction & Weight bearing & $\begin{array}{l}\text { C. Wall push standing on } \\
\text { study leg }\end{array}$ & $\begin{array}{l}\text { Indications: good progression from crab walking (2B) and for } \\
\text { variety at final session }\end{array}$ \\
& & $\begin{array}{l}\text { Progression: Increase step height. Hold extra weight (in hands } \\
\text { or backpack) }\end{array}$ \\
\hline S. Hamstring strengthening (1 exercise) & & $\begin{array}{l}\text { Simplification: If unable to tolerate static standing on joint then } \\
\text { avoid and use 2B or 2A. Precaution in those with increased varus. }\end{array}$ \\
\hline Standing knee flexion $\quad$ Non weight-bearing & $\begin{array}{l}\text { Standing over bench knee } \\
\text { curls with weight }\end{array}$ & $\begin{array}{l}\text { Progression: Increase cuff weight or theraband resistance - red } \\
\text { through to black }\end{array}$ \\
& & Simplification:
\end{tabular}

Simplification: eliminate weight 
Table 2 Home exercise program protocol (Continued)

\begin{tabular}{|c|c|c|c|}
\hline \multicolumn{4}{|c|}{ 4. Calf strengthening (1 exercise) } \\
\hline Standing plantar-flexion & Weight-bearing & Double heel raises & Progression: single heel raises, raises from the edge of a step \\
\hline \multicolumn{4}{|c|}{ 5. Others ( 1 exercise if appropriate) } \\
\hline Knee ROM & Weight bearing & $\begin{array}{l}\text { A. Deep squats holding onto } \\
\text { a bench/chair }\end{array}$ & Progression: increase squat depth \\
\hline Hip ROM & Weight bearing & $\begin{array}{l}\text { B. Deep lunges holding onto } \\
\text { back of chair/bench }\end{array}$ & Progression: increase lunge depth \\
\hline Hip extensors & Weight-bearing & C. Bridging & $\begin{array}{l}\text { Progression: split leg bridge, single bridge with a hold, } \\
\text { bridging one leg }\end{array}$ \\
\hline
\end{tabular}

will be taught how to determine whether pain levels are acceptable or not during and for a short time after the exercises. If a specific exercise is aggravating the participant's pain, then the physiotherapist will reduce the resistance, dosage and/or level of challenge within the exercise until the pain flare settles or change the exercise completely if it remains pain provoking.

\section{Treatment fidelity}

Eight experienced musculoskeletal physiotherapists (4 female, 4 male) in 7 locations around metropolitan Melbourne and regional Victoria have been selected and trained to deliver the study exercise intervention. They have an average of 16 years (minimum 3 years, maximum 28 years) of clinical experience treating musculoskeletal disorders. They attended a full-day training course in Melbourne conducted by the researchers on February 22nd 2014 and were provided with a detailed intervention manual. Training included information about study procedures and protocol, exercise delivery, progression and monitoring. As therapists are encouraged to support and reinforce pain coping skills taught on PainCOACH, they also received background information, exposure and education about those skills so they can be integrated into the exercise program and daily life. Regular telephone/Skype meetings between the physiotherapists and a member of the research team will be held throughout the study. Physiotherapists will complete standardised treatment notes for each participant's individual treatment session and return these via an online survey within 48 hours of the session appointment. Treatment fidelity will be assessed using a treatment check list applied to key points within the treatment notes.

\section{Descriptive data}

Age, gender, duration of knee symptoms, previous treatments, medical history, and medication use will be obtained at baseline by an online questionnaire.

\section{Outcome measures}

The outcome measures are summarised in Table 3. Primary outcomes will be measured at baseline, 3 months and 9 months. Conclusions regarding treatment effectiveness will be evaluated based on changes in primary outcome measures from baseline to 3 months. Our two primary outcomes, which are recommended as valid measures of pain and physical function for knee OA [52], are:

\section{Knee pain on walking measured by an 11-point NRS}

Average knee pain on walking over the past week will be self-reported using an 11-point numeric rating scale (NRS) $(0=$ no pain, $10=$ worst pain possible). This measurement is reliable and valid in OA populations [53]. The minimum clinically important difference to be detected in OA trials has been defined as a change in pain of 1.8 units (out of 10) [54].

\section{Physical function measured by the function subscale of the WOMAC}

Physical function will be measured by the WOMAC Osteoarthritis Index (Likert version 3.1) [55]. This is a disease-specific self-report questionnaire with extensive evidence of validity, reliability and responsiveness in OA populations [56]. The physical function subscale contains 17 questions (each answered on a Likert scale where $0=$ no dysfunction and 4 =extreme dysfunction) and has a total score ranging from 0 (no dysfunction) to 68 (maximum dysfunction). The minimum clinically important difference to be detected in OA trials has been defined as a change in function of 6 points (out of 68) [57].

Our secondary outcome measures, which will collected at baseline, 3 and 9 months, unless otherwise indicated below, include:

\section{Participant-perceived response to treatment measured on 7-point scales}

Participants will rate their perceived change in a) pain, b) physical function and c) overall condition on a sevenpoint ordinal scale (1-much worse to 7 -much better) at 3 and 9 months. This scale will be used as an external criterion for comparison with changes in scores of other outcomes [58]. Measuring participant-perceived change using a rating of change scale has been shown to be a reliable and clinically relevant method of identifying 
Table 3 Summary of outcome measures and time points

\begin{tabular}{|c|c|c|}
\hline Primary outcome measures & Data collection instrument & Collection points \\
\hline Average walking pain in past week & 11-point numeric rating scale & $0,3^{*}$, and 9 months \\
\hline Physical function in past 48 hours & WOMAC osteoarthritis Index physical function subscale & $0,3^{*}$, and 9 months \\
\hline \multicolumn{3}{|l|}{ Secondary outcome measures } \\
\hline Pain in past 48 hours & WOMAC osteoarthritis Index pain subscale & 0,3 and 9 months \\
\hline Perceived change overall & 7-point ordinal scale & 3 and 9 months \\
\hline Perceived change in pain & 7-point ordinal scale & 3 and 9 months \\
\hline Perceived change in function & 7-point ordinal scale & 3 and 9 months \\
\hline Health-related quality of life & Assessment of Quality of Life questionnaire (AQoL2) & 0,3 and 9 months \\
\hline \multirow[t]{3}{*}{ Self-reported psychological measures } & Arthritis self-efficacy scale & 0,3 and 9 months \\
\hline & Coping Strategies questionnaire (CSQ) & 0,3 and 9 months \\
\hline & Pain Catastrophizing Scale (PCS) & 0,3 and 9 months \\
\hline \multicolumn{3}{|l|}{ Other measures } \\
\hline \multirow[t]{8}{*}{ Adherence to intervention } & Number of physiotherapy sessions attended & During intervention \\
\hline & Weekly home exercise log books & During intervention \\
\hline & PainCOACH module completion & During intervention \\
\hline & PainCOACH practice via COACHTrack/ log books & During intervention \\
\hline & Home exercises - 11-point numeric rating scale & 3,6 and 9 months \\
\hline & Frequency of home exercise sessions previous 2 weeks & 3,6 and 9 months \\
\hline & Frequency of pain coping skills in previous 2 weeks & 3,6 and 9 months \\
\hline & Educational material accessed in previous 3 months & 3,6 and 9 months \\
\hline Adverse events and harms & Questionnaire & 3,6 and 9 months \\
\hline Health cost data & Questionnaire & 9 months \\
\hline Descriptive information & Questionnaire & 0 months \\
\hline Medications and co-interventions & Questionnaire & $0,3,9$ months \\
\hline Treatment benefit expectations & 5-point ordinal scale & 0 months \\
\hline \multicolumn{3}{|l|}{ Process measures } \\
\hline Treatment satisfaction & Questionnaire & 3 months \\
\hline Appropriateness of the intervention & Program (System) usability scale & 3 months \\
\hline
\end{tabular}

${ }^{¥}$ Primary outcomes and primary time point.

improvements that are truly meaningful from the individual perspective [59]. Participants who report that they are moderately better and above will be classified as improved.

\section{Knee pain measured by the WOMAC pain subscale}

Knee pain will also be captured using the WOMAC pain subscale, which contains five questions, each answered on a Likert scale where $0=$ no pain and $4=$ extreme pain. It has a total score ranging from 0 (no pain) to 20 (maximum pain) [55].

\section{Health-related quality of life measured by the Assessment of Quality of Life (AQoL)}

The AQoL questionnaire (version AQoL-II) contains 20 questions that cover six dimensions of health-related quality of life including independent living, social relationships, physical senses, coping, pain and psychological wellbeing. Responses to each question are answered on 5 -point scale. Scores range from -0.04 (worst possible health-related quality of life) to 1.00 (full health-related quality of life). The AQoL-II has strong evidence of validity and responsiveness [60,61]. A clinically important difference in health-related quality of life has been defined as a change of 0.04 AQoL units [62].

\section{Self-efficacy for pain and function measured by the arthritis self-efficacy scale}

Self-efficacy for pain management and its effects on function will be measured with the Arthritis Self-Efficacy Scale [63], which assess confidence for managing pain (5 questions) and physical function (9 questions) on a 10-point NRS (where 1 = very uncertain and $10=$ very certain). Responses are averaged so that higher scores indicate greater self- 
efficacy. This scale is reliable and valid in OA populations [63].

\section{Pain catastrophising using the pain catastrophising scale} The Pain Catastrophising Scale measures the tendency to ruminate about pain, magnify pain, and feel helpless about pain. All 13 items are measured on a 5-point Likert scale (where $0=$ low levels of catastrophising and $4=$ high levels of catastrophising). The highest possible total score of 52 indicates the greatest level of catastrophising. It has high internal consistency and is associated with heightened pain, psychological distress, and physical disability [64].

\section{Use of coping skills to manage pain using the coping} attempts scale of the Coping Strategies Questionnaire (CSQ) The CSQ [65] can be used to measure how often a patient uses six cognitive and behavioural pain coping strategies to manage pain (diverting attention, reinterpreting pain sensations, coping self-statements, ignoring pain sensations, praying and hoping, and increasing activity level). Items are measured on a 7-point Likert scale (where $0=$ never uses coping skills and $7=$ always uses coping skills). Based on prior factor analyses of this instrument [66], participant's responses will be converted to scores on the Coping Attempts factor of the CSQ. The CSQ has demonstrated sensitivity to change from treatment in chronic pain samples as well as good internal consistency and construct validity [66].

\section{Other measures}

\section{Treatment adherence}

The number of Skype physiotherapy sessions attended by each participant will be recorded by the treating physiotherapist and submitted to the researchers using scheduled online treatment notes. The number of Pain$\mathrm{COACH}$ sessions completed will be automatically recorded by the program progress log which will be monitored regularly by the researchers. During the intervention period, adherence to the home exercise program will be self-reported in weekly log books, which at the completion of the intervention will either be transcribed to an online survey or returned by post, depending on participant preference. Pain coping skills practice and coping confidence (self-efficacy for using pain coping skills to manage pain) will be recorded using the Pain$\mathrm{COACH}$ practice tracking log (COACHtrack) or alternatively by completing weekly logs that will either be transcribed to an online survey or returned by post.

Participants in the PCST + Ex group will also rate their adherence to their home exercise program over the previous 3 months (from 'not at all' to 'completely as instructed') at 3, 6 and 9 months using an online 11-point NRS. They will also report the number of exercise sessions they completed and how often they used the PainCOACH skills in the previous 2 weeks, at 3, 6, and 9 months. Participants in both groups will report which online education information sheets were accessed in the past 3 months 3, 6, and 9 months using an online checklist.

\section{Adverse events and use of health services/co-interventions}

Adverse events will be defined as any health problems that participants: i) believed to be caused by the treatment; ii) that required them to see a health professional or take medications; iii) and/or that interfered with function for 2 or more days. Information on adverse events throughout the 13 week intervention will be collected for the combined group via exercise log books and at 3 , 6 and 9 months for both groups via an online survey. Participants' use of health services and co-interventions (medication for knee pain and any other treatment for knee pain) will be collected via an online survey at 0,3 and 9 months. Direct health care costs and direct nonhealth care resources will be collected at the 9 months via an online survey.

\section{Expectations of treatment effect}

At baseline, participants will be asked about their expectations of the effect they think the study treatments will have on their knee pain using a 5-point ordinal scale ranging from no effect to complete recovery.

\section{Process measures}

Additional process measures will be collected for both groups at 3 months for the purposes of measuring the acceptability of the interventions (rather than to determine treatment efficacy). These additional measures include: i) appropriateness of the interventions using a custom designed questionnaire and ii) satisfaction with the intervention using a 2-item custom designed questionnaire that asks about the overall satisfaction with the content and delivery of the intervention.

\section{Sample size}

The primary endpoints of the trial are between-group (PCST + Ex group versus the educational control group) differences in change in knee pain on walking (NRS) and change in physical function (WOMAC). Moderate betweengroup treatment effects of around 0.5 have previously been reported for pain and function following individual exercise programs in people with knee OA $[13,67]$. Therefore, to enable detection of at least a 0.5 effect size between groups in either pain or function in a two-arm trial with $80 \%$ power, a significance level at 0.05 and allowing for $15 \%$ attrition rate, we will recruit 74 participants in each group or a total of 148 participants. 


\section{Data and statistical analysis}

A biostatistician (MPS) blinded to group allocation will analyse data. Main comparative analyses between groups will be performed using intention-to-treat. This analysis will include all participants who have missing data and those who do not fully adhere to the protocol. Demographic characteristics, as well as baseline scores on primary and secondary outcome measures, will be inspected to assess baseline comparability of treatment groups. These variables will also be examined for those participants who withdraw from the study and those who remain. Descriptive statistics will be presented for each group as the mean change (standard deviation, 95\% confidence intervals) in the outcomes from baseline to each time-point. For continuous outcomes differences in mean change will be compared between groups using linear regression random effects modelling adjusted for baseline values of the outcome. Proportional odds models will compare improvement between groups based on perceived ratings of change as well as the proportion in each group who attain the minimum clinical important difference (MCID) for pain and function. The MCID to be detected in OA trials is a change in pain of 1.8 units (out of 10) [54] and change in function of 6 points (out of 68) [57]. Model diagnostic checks will utilise residual plots. Similar regression models for binary and ordinal outcome measures will use random effects logistic and proportional odds models, respectively. We will also perform a per protocol analysis as appropriate.

\section{Timelines}

Ethics approval was obtained from the Human Research Ethics Committee of the University of Melbourne in August 2013. Recruitment and training of the physiotherapists was completed during February 2014. The trial was registered with the Australian and Zealand Clinical Trials Registry reference: ACTRN12614000243617 in March 2014 prior to participant recruitment which commenced in March 2014 and is expected to be completed by June 2015. The trial is due for completion in March 2016 when all participants are expected to have completed the 9-month follow up.

\section{Discussion}

This paper provides the justification and the protocol for a pragmatic RCT that will investigate whether Internetbased PCST + Ex is more effective than online educational material alone in people with persistent knee pain consistent with a clinical diagnosis of knee OA. The findings of this study will help determine if a PCST and exercise intervention that is delivered using the Internet over 3 months can improve self-reported pain and physical function when compared to an educational control.
Identification of feasible healthcare models that improve outcomes in people with persistent knee pain and knee OA has important implications for clinical practice. Our study will provide new information about the effectiveness and acceptability of a novel clinical practice model for the delivery of interventions that are known to be beneficial to people with persistent knee pain. Findings could therefore have the potential to guide clinical practice towards innovative modes of healthcare provision. This does not diminish the importance of usual methods of delivery of health care, such as face-to-face visits when access is available, however this model could be of particular benefit to people in regional and remote areas who may not be able to access face-to-face care.

In order to maximise translatability, this study was intentionally designed to test a service delivery model that could be easily implemented. Use of a limited number of physiotherapy contacts similar to usual care, a home-based exercise program, and the Internet as a vehicle to deliver the intervention, which is becoming more widely accessible and acceptable to this patient population, will assist this endeavour. If the results of our proposed study support the benefits of Internetbased PCST + Ex, the components of this intervention could be easily implemented into clinical practice.

Strengths of this RCT study design are the pragmatic nature of treatment delivery by practising physiotherapists as well as the reproducibility of both the physiotherapy and pain coping skills programs. These features will improve the ability to translate the findings into a range of health care settings and enable future researchers to replicate the intervention. Importantly, the physiotherapy-guided home exercise program is individualised with regard to the content, and the intensity level of the exercises are individually monitored, progressed or stepped back throughout the intervention according to participant feedback. Additionally, physiotherapists will support and reinforce the participants' use of the pain coping skills during the exercise sessions so as these skills can not only be integrated into the exercise program, but also encouraged within daily life. The inclusion of participants regardless of their geographic location will further enhance the generalisability of results. The study is adequately powered for our primary outcome measures.

\section{Abbreviations}

AQoL: Assessment of quality of life; CONSORT: Consolidated standards of reporting trials; CSQ: Coping strategies questionnaire; MCID: Minimal clinical important difference; NRS: Numeric rating scale; OA: Osteoarthritis; PCST: Pain coping skills training; RCT: Randomised controlled trial; WOMAC: Western Ontario McMaster Universities osteoarthritis index.

\section{Competing interests}

The Internet-based pain coping skills program (PainCOACH) which will be used in this study was co-developed by two of the study investigators 
(FK, CR). The authors declare that they have no other competing interests.

\section{Authors' contributions}

$\mathrm{KLB}, \mathrm{FK}, \mathrm{CR}$, and $\mathrm{RSH}$ conceived the project and $\mathrm{KLB}$ is leading the trial. $\mathrm{KLB}$ procured the project funding. $\mathrm{KLB}, \mathrm{FK}, \mathrm{CR}, \mathrm{RSH}, \mathrm{JHA}$ and FD developed the protocol. KLB, RSH, FD and $A D$ designed the physiotherapy program and $A D$, FD and $C B$ trained the physiotherapists. RN co-ordinates the trial, recruits and screens the participants. FD wrote the first and final draft of this manuscript. MPS performed the sample size calculations and designed the randomisation schedule and statistical analyses. All authors participated in the trial design, provided feedback on drafts of this paper and read and approved the final manuscript.

\section{Acknowledgements}

This trial is being funded by the National Health and Medical Research Council (Program Grant \#631717). None of the funders have any role in the study other than to provide funding. KLB is funded by a National Health and Medical Research Council Principal Research Fellowship (\#1058440). RSH is funded by an Australian Research Council Future Fellowship (FTFT0991413). JHA is funded by a Sir Charles Hercus Health Research Fellowship from the Health Research Council of New Zealand.

\section{Author details}

${ }^{1}$ Centre for Health, Exercise and Sports Medicine, Department of Physiotherapy, School of Health Sciences, The University of Melbourne, Alan Gilbert Building 161 Barry St, Carlton, Vic 3053, Australia. ${ }^{2}$ School of Rehabilitation Therapy, Faculty of Health Sciences, Queen's University, Kingston, Ontario, Canada. ${ }^{3}$ Gillings School of Global Public Health, Department of Health Behavior, University of North Carolina, Chapel Hill, NC, Raleigh, USA. ${ }^{4}$ Department of Psychiatry and Behavioral Sciences, Duke University, Durham, NC, USA. ${ }^{5}$ Centre for Musculoskeletal Outcomes Research, Department of Surgical Sciences, Dunedin School of Medicine, University of Otago, Dunedin, New Zealand. ${ }^{6}$ Psychological Sciences, University of Melbourne and Royal Women's Hospital, Melbourne, Vic, Australia. ${ }^{7}$ Department of Epidemiology at Cabrini, Cabrini Institute and Monash University, Malvern, Victoria, USA. ${ }^{8}$ Physioworks Health Group, Camberwell, Victoria, Australia.

Received: 31 July 2014 Accepted: 8 August 2014 Published: 13 August 2014

\section{References}

1. Felson DT, Lawrence RC, Dieppe PA, Hirsch R, Helmick CG, Jordan JM, Kington RS, Lane NE, Nevitt MC, Zhang Y, Sowers M, McAlindon T, Spector TD, Poole AR, Yanovski SZ, Ateshian G, Sharma L, Buckwalter JA, Brandt KD, Fries JF: Osteoarthritis: new insights. Part 1: the disease and its risk factors. Ann Intern Med 2000, 133(8):635-646.

2. Hoy DG, Smith E, Cross M, Sanchez-Riera L, Buchbinder R, Blyth FM, Brooks P, Woolf AD, Osborne RH, Fransen M, Driscoll T, Vos T, Blore JD, Murray C, Johns N, Naghavi M, Carnahan E, March LM: The global burden of musculoskeletal conditions for 2010: an overview of methods. Ann Rheum Dis 2014, 73(6):982-989.

3. Cross M, Smith E, Hoy D, Nolte $S$, Ackerman I, Fransen M, Bridgett L, Williams S, Guillemin F, Hill CL, Laslett LL, Jones G, Cicuttini F, Osborne R, Vos T, Buchbinder R, Woolf A, March L: The global burden of hip and knee osteoarthritis: estimates from the global burden of disease 2010 study. Ann Rheum Dis 2014, 73(7):1323-1330.

4. Salaffi F, Carotti M, Stancati A, Grassi W: Health-related quality of life in older adults with symptomatic hip and knee osteoarthritis: a comparison with matched healthy controls. Aging Clin Exp Res 2005, 17(4):255-263.

5. Scopaz K, Piva S, Wisniewski S, Fitzgerald G: Relationships of fear, anxiety, and depression with physical function in patients with knee osteoarthritis. Arch Phys Med Rehabil 2009, 90:1866-1873.

6. Brosseau L, Pelland L, Wells G, Macleay L, Lamothe C, Michaid G, Lambert J, Robinson V, Tugwell P: Efficacy of aerobic exercises for osteoarthritis (Part II): a meta-analysis. Phys Ther Rev 2004, 9:125-145.

7. Pelland L, Brosseau L, Wells G, MacLeay L, Lambert J, Lamothe C, Robinson V, Tugwell P: Efficacy of strengthening exercises for osteoarthritis (Part I): a meta-analysis. Phys Ther Rev 2004, 9:77-108.
8. Lange AK, Vanwanseele B, Singh MAF: Strength training for treatment of osteoarthritis of the knee: a systematic review. Arthritis Rheum 2008, 59(10):1488-1494.

9. Lim BW, Hinman RS, Wrigley TV, Bennell KL: Varus malalignment and its association with impairments and functional limitations in medial knee osteoarthritis. Arthritis Rheum 2008, 59(7):935-942.

10. Bennell KL, Hunt MA, Wrigley TV, Hunter DJ, McManus FJ, Hodges PW, Li L, Hinman RS: Hip strengthening reduces symptoms but not knee load in people with medial knee osteoarthritis and varus malalignment: a randomised controlled trial. Osteoarthritis Cartilage 2010, 18(5):621-628.

11. Nelson AE, Allen KD, Golightly YM, Goode AP, Jordan JM: A systematic review of recommendations and guidelines for the management of osteoarthritis: the chronic osteoarthritis management initiative of the U.S. bone and joint initiative. Semin Arthritis Rheum 2013, Epub 2014/01/07.

12. Juhl C, Christensen R, Roos EM, Zhang W, Lund H: Impact of exercise type and dose on pain and disability in knee osteoarthritis: a systematic review and meta-regression analysis of randomized controlled trials. Arthritis Rheum 2014, 66(3):622-636

13. Harmer AR, McConnell S, Simic M, van der Esch M, Bennell KL, Fransen M: Sustainability of effects of land-based exercise on pain and physical function for osteoarthritis of the knee: systematic review and meta-analysis. Osteoarthritis Cartilage 2014, 22(Supplement(0)):S388.

14. Uthman OA, van der Windt DA, Jordan JL, Dziedzic KS, Healey EL, Peat GM, Foster NE: Exercise for lower limb osteoarthritis: systematic review incorporating trial sequential analysis and network meta-analysis. BMJ 2013, 347:f5555.

15. Roddy E, Zhang W, Doherty M, Arden N, Barlow J, Birrell F, Carr A, Chakravarty K, Dickson J, Hay E, Hosie G, Hurley M, Jordan K, McCarthy C, McMurdo M, Mockett S, O'Reilly S, Peat G, Pendleton A, Richards S: Evidence-based recommendations for the role of exercise in the management of osteoarthritis of the hip or knee- the MOVE consensus. Rheumatology 2005, 44(1):67-73.

16. Bennell KL, Hinman RS: A review of the clinical evidence for exercise in osteoarthritis of the hip and knee. J Sci Med Sport 2011, 14(1):4-9.

17. Fransen M, McConnell S: Exercise for osteoarthritis of the knee. Cochrane Database Syst Rev 2008, 4, CD004376.

18. Sale JE, Gignac M, Hawker G: The relationship between disease symptoms, life events, coping and treatment, and depression among older adults with osteoarthritis. J Rheumatol 2008, 35(2):335-342.

19. Keefe F, Bonk V: Psychosocial assessment of pain in patients having rheumatic diseases. Rheum Dis Clin North Am 1999, 25:81-103.

20. Dixon KE, Keefe FJ, Scipio CD, Perri LM, Abernethy AP: Psychological interventions for arthritis pain management in adults: a meta-analysis. Health Psychol 2007, 26(3):241-250.

21. Devos-Comby L, Cronan T, Roesch SC: Do exercise and self-management interventions benefit patients with osteoarthritis of the knee? A metaanalytic review. J Rheumatol 2006, 33(4):744-756.

22. Hurley MV, Walsh NE: Effectiveness and clinical applicability of integrated rehabilitation programs for knee osteoarthritis. Curr Opin Rheumatol 2009, 21(2):171-176.

23. Keefe FJ, Blumenthal J, Baucom D, Affleck G, Waugh R, Caldwell DS, Beaupre P, Kashikar-Zuck S, Wright K, Egert J, Lefebvre J: Effects of spouse-assisted coping skills training and exercise training in patients with osteoarthritic knee pain: a randomized controlled study. Pain 2004, 110(3):539-549.

24. Hunt MA, Keefe FJ, Bryant C, Metcalf BR, Ahamed Y, Nicholas MK, Bennell KL: A physiotherapist-delivered, combined exercise and pain coping skills training intervention for individuals with knee osteoarthritis: a pilot study. Knee 2013, 20(2):106-112.

25. Badley E, DesMeules M: Arthritis in Canada: an ongoing challange. Ottawa (Canada): Health Canada; 2003.

26. Clauw DJ: Pain management: fibromyalgia drugs are 'as good as it gets' in chronic pain. Nat Rev Rheumatol 2010, 6(8):439-440.

27. Marcus $\mathrm{BH}$, Ciccolo JT, Sciamanna CN: Using electronic/computer interventions to promote physical activity. Br J Sports Med 2009, 43(2):102-105.

28. Internet World Stats: Usage and Population Statistics. Available from: [http://www.internetworldstats.com/stats.htm]. 2012, Accessed 23/06/2014.

29. Zickuhr K, Madden M: Older Adults and Internet Use. In Pew Research Center's Internet \& American Life Project; 2012.

30. Strecher $\mathrm{V}$ : Internet methods for delivering behavioral and health-related interventions (eHealth). Annu Rev Clin Psychol 2007, 3:53-76. 
31. Rini C, Williams DA, Broderick JE, Keefe FJ: Meeting them where they are: using the internet to deliver behavioral medicine interventions for pain. Transl Behav Med 2012, 2(1):82-92.

32. Macea DD, Gajos K, Daglia Calil YA, Fregni F: The efficacy of web-based cognitive behavioral interventions for chronic pain: a systematic review and meta-analysis. J Pain 2010, 11(10):917-929.

33. Cuijpers P, Straten A, Andersson G: Internet-administered cognitive behavior therapy for health problems: a systematic review. J Behav Med 2007, 31(2):169-177.

34. Pietrzak E, Cotea C, Pullman S, Nasveld P: Self-management and rehabilitation in osteoarthritis: is there a place for internet-based interventions? Telemed J E Health 2013, 19(10):800-805.

35. Wong YK, Hui E, Woo J: A community-based exercise programme for older persons with knee pain using telemedicine. J Telemed Telecare 2005 11(6):310-315

36. Russell TG, Buttrum P, Wootton R, Jull GA: Internet-based outpatient telerehabilitation for patients following total knee arthroplasty: a randomized controlled trial. J Bone Joint Surg 2011, 93(2):113-120.

37. Tousignant M, Moffet $H$, Boissy P, Corriveau H, Cabana F, Marquis F: A randomized controlled trial of home telerehabilitation for post-knee arthroplasty. J Telemed Telecare 2011, 17(4):195-198.

38. Tousignant M, Boissy P, Moffet H, Corriveau H, Cabana F, Marquis F, Simard J: Patients' satisfaction of healthcare services and perception with in-home telerehabilitation and physiotherapists' satisfaction toward technology for post-knee arthroplasty: an embedded study in a randomized trial. Telemed J E Health 2011, 17(5):376-382.

39. Boutron I, Moher D, Altman DG, Schulz KF, Ravaud P: Extending the CONSORT statement to randomized trials of nonpharmacologic treatment: explanation and elaboration. Ann Intern Med 2008, 148(4):295-309.

40. Schulz KF, Altman DG, Moher D: CONSORT 2010 statement: updated guidelines for reporting parallel group randomized trials. Ann Intern Med 2010, 152(11):726-732.

41. Keefe FJ, Beaupre PM, Gil KM, Rumble ME, Aspnes AK: Group Therapy for Patients with Chronic Pain. In Pain Management: A Practitioner's Handbook. 2nd edition. Edited by Turk DC, Gatchel RJ. New York: Guilford Press; 2002:234-255

42. Melzack R, Wall P: Pain mechanisms: a new theory. Science 1965, 150:971-979

43. Rini C, Porter LS, Somers TJ, McKee DC, Keefe FJ: Retaining critical therapeutic elements of behavioral interventions translated for delivery via the internet: recommendations and an example using pain coping skills training. J Med Internet Res Under revision.

44. Bandura A: Self-efficacy: The exercise of Control. New York: W.H. Freeman and Company; 1997

45. Bandura A: Social Foundations of Thought and Action: A Social Cognitive Theory. Englewood Cliffs, N.J.: Prentice-Hall; 1986.

46. Knowles MS: The Adult Learner: A Neglected Species. 3rd edition. Houston: Gulf Pub. Co., Book Division; 1984.

47. Knowles MS, Holton EF, Swanson RA: The Adult Learner: The Definitive Classic in Adult Education and Human Resource Development. 7th edition. Boston: Elsevier; 2011.

48. Mayer RE: Applying the science of learning: evidence-based principles for the design of multimedia instruction. Am Psychol 2008, 63(8):760-769.

49. Bennell KL, Egerton T, Bills C, Gale J, Kolt GS, Bunker SJ, Hunter DJ, Brand CA, Forbes A, Harris A, Hinman RS: Addition of telephone coaching to a physiotherapist-delivered physical activity program in people with knee osteoarthritis: a randomised controlled trial protocol. BMC Musculoskelet Disord 2012, 13:246.

50. Fransen M, McConnell S: Exercise for Osteoarthritis of the Knee. Cochrane Database Syst Rev 2008, 4:CD004376.

51. Day ML, McGuigan MR, Brice G, Foster C: Monitoring exercise intensity during resistance training using the session RPE scale. J Strength Cond Res 2004, 18(2):353-358.

52. Bellamy N: Outcome measurement in osteoarthritis clinical trials. J Rheumatol 1995, 22:49-51.

53. Bellamy N: Osteoarthritis clinical trials: candidate variables and clinimetric properties. J Rheumatol 1997, 24(4):768-778.

54. Bellamy N, Carette S, Ford P, Kean W, le Riche N, Lussier A, Wells G, Campbell J: Osteoarthritis antirheumatic drug trials. III. Setting the delta for clinical trials- results of a consensus development (Delphi) exercise. J Rheumatol 1992, 19(3):451-457.
55. Bellamy N, Buchanan WW, Goldsmith CH, Campbell J, Stitt LW: Validation study of WOMAC: a health status instrument for measuring clinically important patient relevant outcomes to antirheumatic drug therapy in patients with osteoarthritis of the hip or knee. J Rheumatol 1988, 15(12):1833-1840.

56. McConnell S, Kolopack P, Davis AM: The Western Ontario and McMaster Universities Osteoarthritis Index (WOMAC): a review of its utility and measurement properties. Arthritis Rheum 2001, 45(5):453-461.

57. Tubach F, Ravaud P, Baron G, Falissard B, Logeart I, Bellamy N, Bombardier C, Felson D, Hochberg M, van der Heijde D, Dougados M: Evaluation of clinically relevant changes in patient reported outcomes in knee and hip osteoarthritis: the minimal clinically important improvement. Ann Rheum Dis 2005, 64(1):29-33.

58. Jaeschke R, Singer J, Guyatt GH: Measurement of health status: ascertaining the minimal clinically important difference. Control Clin Trials 1989, 10(4):407-415.

59. ten Klooster PM, Drossaers-Bakker KW, Taal E, van de Laar MA: Patientperceived satisfactory improvement (PPSI): interpreting meaningful change in pain from the patient's perspective. Pain 2006, 121(1-2):151-157.

60. Whitfield K, Buchbinder R, Segal L, Osborne RH: Parsimonious and efficient assessment of health-related quality of life in osteoarthritis research: validation of the Assessment of Quality of Life (AQoL) instrument. Health Qual Life Outcomes 2006, 4:19.

61. Osborne RH, Hawthorne G, Lew EA, Gray LC: Quality of life assessment in the community-dwelling elderly: validation of the Assessment of Quality of Life (AQoL) Instrument and comparison with the SF-36. J Clin Epidemiol 2003, 56(2):138-147

62. Hawthorne G, Osborne R: Population norms and meaningful differences for the Assessment of Quality of Life (AQoL) measure. Aust N Z J Public Health 2005, 29(2):136-142.

63. Lorig K, Chastain RL, Ung E, Shoor S, Holman HR: Development and evaluation of a scale to measure perceived self-efficacy in people with arthritis. Arthritis Rheum 1989, 32(1):37-44.

64. Osman A, Barrios FX, Gutierrez PM, Kopper BA, Merrifield T, Grittmann L: The pain catastrophizing scale: further psychometric evaluation with adult samples. J Behav Med 2000, 23(4):351-365.

65. Rosenstiel AK, Keefe FJ: The use of coping strategies in chronic low back pain patients: relationship to patient characteristics and current adjustment. Pain 1983, 17(1):33-44.

66. Keefe FJ, Caldwell DS, Queen KT, Gil KM, Martinez S, Crisson JE, Ogden W, Nunley J: Pain coping strategies in osteoarthritis patients. J Consult Clin Psychol 1987, 55(2):208-212.

67. Fransen M, McConnell S: Land-based exercise for osteoarthritis of the knee: a meta-analysis of randomized controlled trials. J Rheumatol 2009, 36(6):1109-1117.

\section{doi:10.1186/1471-2474-15-279}

Cite this article as: Dobson et al:: Internet-mediated physiotherapy and pain coping skills training for people with persistent knee pain (IMPACT - knee pain): a randomised controlled trial protocol. BMC Musculoskeletal Disorders 2014 15:279.

\section{Submit your next manuscript to BioMed Central and take full advantage of:}

- Convenient online submission

- Thorough peer review

- No space constraints or color figure charges

- Immediate publication on acceptance

- Inclusion in PubMed, CAS, Scopus and Google Scholar

- Research which is freely available for redistribution 